\title{
Velocidad de migración de las células de músculo liso vascular de ratas Wistar
}

\author{
Montoya Azpeitia, Stephanie Gisel*, Mejía-Elizondo, Rebeca**, Tanguma-Espinosa, Ricardo**
}

\begin{tabular}{|c|c|}
\hline $\begin{array}{l}\text { Resumen } \\
\text { Introducción: La diabetes mellitus es una de las enfermeda- } \\
\text { des con mayor impacto sociosanitario, no sólo por su alta } \\
\text { prevalencia, sino también por las complicaciones que pro- } \\
\text { duce, los factores de riesgo que implica y por su elevada } \\
\text { tasa de mortalidad. Actualmente existen } 347 \text { millones de } \\
\text { personas con esta enfermedad en el mundo. Las complica- } \\
\text { ciones macrovasculares aumentan la morbimortalidad car- } \\
\text { diovascular, constituyendo la principal causa de muerte en } \\
\text { los diabéticos. Eso involucra el proceso de ateroesclerosis y } \\
\text { su evolución puede llevar a un infarto siendo necesaria para } \\
\text { el tratamiento la implantación de una endoprótesis vascular; } \\
\text { sin embargo, éstas tienen un alto porcentaje de re-estenosis, } \\
\text { debido a la migración de células de músculo liso vascular } \\
\text { (CMLV), por lo que resalta la importancia del estudio de } \\
\text { dicho fenómeno. Objetivos: La medición de la velocidad de } \\
\text { migración de las CMLV de aorta de ratas macho Wistar, } \\
\text { control y diabética inducida. Metodología: Modelo de estu- } \\
\text { dio experimental, transversal y comparativo. Las variables } \\
\text { de respuesta fueron: el porcentaje de migración de CMLV } \\
\text { en unidades arbitrarias mediante el experimento "herida- } \\
\text { cicatrización" en caja de cultivo. Resultados: Se registraron } \\
\text { porcentajes promedio de velocidad de migración de CMLV a } \\
\text { las I } 2 \text { horas de } 20.6 \% \text { y } 47.28 \% \text {; mientras que a las } 24 \text { ho- } \\
\text { ras de } 5 \text { I.8\% y de } 81.87 \% \text { para las ratas control y diabética } \\
\text { respectivamente. Conclusiones: La velocidad de migración de } \\
\text { las CMLV es mayor en las células de rata diabética que } \\
\text { la de rata control. LuXMÉDICA AÑ011, NúmER034, SEPTIEMBRE- } \\
\text { DICIEMBRE2016,PP63-70 }\end{array}$ & $\begin{array}{l}\text { Abstract } \\
\text { Introduction: Diabetes is a disease with one of the largest } \\
\text { impacts on public health: not only because of its high pre- } \\
\text { valence, but also by the complications that occurs, the risk } \\
\text { factors involved and by its high rate of mortality. Currently } \\
\text { there are } 347 \text { million people with this disease in the world. } \\
\text { Macrovascular complications increase the cardiovascular } \\
\text { morbimortality, constitute the leading cause of death in dia- } \\
\text { betics. That involves the process of atherosclerosis and its } \\
\text { evolution can lead to a heart attack being necessary for } \\
\text { treating a vascular stent implantation, however, these have } \\
\text { a high percentage of re-estenosis, due to the migration of } \\
\text { vascular smooth muscle cells, so it emphasizes the impor- } \\
\text { tance of the study of this phenomenon. Objectives:The mea- } \\
\text { surement of the speed of migration of the aorta vascular } \\
\text { smooth muscle cells of male Wistar rats, induced diabetic } \\
\text { and control. Methodology: model of experimental, descrip- } \\
\text { tive, cross-sectional and comparative study. The response } \\
\text { variables were: percentage of vascular smooth muscle cells } \\
\text { migration in arbitrary units through the "wound-healing" } \\
\text { experiment in culture box. Results: Average percentage of } \\
\text { vascular smooth muscle cells migration speed were recorded } \\
\text { at } 12 \text { o'clock of } 20.6 \% \text { and } 47.28 \% \text {; While at } 24 \text { hours of } \\
51.8 \% \text { and } 81.87 \% \text { for rats control and diabetic respectively. } \\
\text { Conclusions: The speed of migration of the vascular smooth } \\
\text { muscle cells is greater in cells of diabetic rat than the rat- } \\
\text { control.LUXmÉDICA AÑ011, NúmER0 34, SEPTIEMBRE-DICIEMBRE } \\
\mathbf{2 0 1 6 , P P ~} 63-70\end{array}$ \\
\hline $\begin{array}{r}\text { Palabras clave: Diabetes, stent, músculo liso vascular, } \\
\text { migración celular. }\end{array}$ & $\begin{array}{l}\text { Key words: Diabetes, Stent, vascular smooth muscle, cell } \\
\text { migration. }\end{array}$ \\
\hline
\end{tabular}

* Estudiante de octavo semestre de la carrera de Médico Cirujano del Centro de Ciencias de la Salud de la Universidad Autónoma de Aguascalien-
tes., Aguascalientes, Ags. México. ** Departamento de Fisiología, Facultad de Medicina de la Universidad Autónoma de San Luis Potosí, SLP, México.

Fecha de recibido: 10 de agosto 2016

Fecha de aceptación: 20 de septiembre 2016

Correspondencia: Dra Rebeca Mejía Elizondo. Departamento de Fisiología, Facultad de Medicina de la Universidad Autónoma de San Luis Potosí, Av Venustiano Carranza No. 2405, Código Postal 78210, San Luis Potosí, S.LP., México. Correo electrónico rebe_mejia@hotmail.com 


\section{Introducción}

La diabetes mellitus (DM) es un grupo complejo de trastornos metabólicos, que en común comparten hiperglucemia, y el déficit de insulina, ya sea por disminución en su producción, secreción o aumento en la resistencia a la misma. ${ }^{1}$ Las últimas estimaciones indican una prevalencia global de 382 millones de personas con diabetes en 2013, se espera que aumente a 592 millones para 2035. ${ }^{2}$ La DM es una de las enfermedades con mayor impacto socio-sanitario, no sólo por su alta prevalencia, sino también por las complicaciones que produce, los factores de riesgo que implica y por su elevada tasa de mortalidad. ${ }^{3}$ Las complicaciones crónicas de la diabetes se clasifican en: a) macrovasculares (equivalente a ateriosclerosis), que son las que afectan a las arterias en general produciendo enfermedad cardíaca coronaria, cerebrovascular y vascular periférica; b) microvasculares, que incluiría la retinopatía, nefropatía y neuropatía, y c) el pie diabético, que aparecería como consecuencia de la neuropatía y/o de la vascular de origen macroangiopático. ${ }^{4}$ Las repercusiones de las complicaciones macrovasculares comportan un incremento de 3 a 4 veces en la morbimortalidad cardiovascular, constituyendo la principal causa de muerte en los diabéticos. ${ }^{5}$ Más del $65 \%$ de los pacientes con DM, mueren por enfermedad cardiovascular (cardiopatía isquémica, cardiopatía hipertensiva, ateroesclerosis) o enfermedad cerebral vascular. ${ }^{6}$

Como consecuencia y evolución del proceso de ateroesclerosis, dentro de las complicaciones macrovasculares, se observa cómo la placa ateroesclerosa endurece y estrecha las arterias, lo cual limita el flujo de sangre rica en oxígeno al tejido que irriga la arteria comprometida; hablando del miocardio puede traducirse esto como isquemia, lesión o infarto. ${ }^{7}$ Al producirse un infarto, se obliga a emplear terapéuticas de revascularización coronaria, como la angioplastía coronaria y la colocación de stent (endoprótesis vascular). ${ }^{8}$ Este procedimiento suele sufrir un proceso de re-estenosis en alrededor del $28 \%$ de los casos. Se ha documentado que es debido a una proliferación excesiva de células de músculo liso vascular estimuladas por el factor de crecimiento derivado de las plaquetas (PDGF), insulina e hiperglucemia. ${ }^{9-11}$

Los factores de riesgo que favorecen una re-estenosis son el síndrome metabólico (hipertrigliceridemia, hiperinsulinemia), diabetes mellitus (hiperglucemia), una mayor longitud de la lesión tratada, menor diámetro de referencia del vaso anterior y un menor diámetro luminal al final del procedimiento. ${ }^{12}$ Durante el proceso de ateroesclerosis, las células de músculo liso vascular cambian su fenotipo contráctil a uno sintético, adquiriendo la capacidad de proliferar y migrar. La migración es un componente importante en el proceso de remodelación vascular, ${ }^{13,14}$ es un ciclo que inicia con la polarización de la célula, como respuesta a un estímulo quimiotáctico, se conoce que es un fenómeno dependiente de calcio. ${ }^{15}$ 
Las células presentan un gradiente de concentración de $\mathrm{Ca}^{2+}$ entre el polo posterior y anterior, siendo mayor en el polo posterior, si el calcio es quelado, el gradiente se abole y la migración se detiene. ${ }^{16}$ Para que la migración celular pueda llevarse a cabo indispensablemente se requieren aumentos transitorios en la concentración de calcio, este aumento inicia en el polo posterior y luego se dirige hacia el polo anterior, formando ondas de $\mathrm{Ca}^{2+} .{ }^{17}$ Se ha encontrado que al inhibir SERCA (ATPasa del retículo sarco endoplásmico) estas ondas se abolen por lo que se deduce que éstas tienen su origen en el retículo sarco endoplásmico. ${ }^{17}$

En la diabetes mellitus existe un estado de estrés del retículo sarco endoplásmico que se asocia a una expresión disminuida de SERCA, y por ende disminuye la concentración de $\mathrm{Ca}^{2+}$ almacenado. Bajo esta información, se supondría que no habría suficiente $\mathrm{Ca}^{2+}$ disponible para el proceso de migración. Sin embargo, esta información además de contrastar con lo anterior expuesto sobre el papel esencial del $\mathrm{Ca}^{2+}$, no explica el proceso de la migración descontrolada de las células del músculo liso vascular en la diabetes mellitus. Existe evidencias de que el músculo liso vascular de ratas diabéticas muestra un estado de hiperreactividad vascular, asociado a la disminución de la expresión de SERCA, y se postula que a pesar de no existir las concentraciones adecuadas de $\mathrm{Ca}^{2+}$ para producir las ondas, existiría el suficiente para que se dé el proceso de migración. ${ }^{17,18}$ El objetivo de este trabajo fue comparar la velocidad de migración de las células de músculo liso vascular (CMLV) entre rata Wistar macho diabética y rata control.

\section{| | | | | | | | | | | | | | | | | | | | | | | | | | | | | | | | | | | | | | | | | | | | | | | | | | | | | | | | | | | | | | | | | | | | | | | | | | | | | | | | | | | | | | | | | | | | | | | | | | | | | | ||}

\section{Material y métodos}

Se trata de un modelo de estudio experimental, transversal y comparativo. Las variables de respuesta fueron: el porcentaje de migración de músculo liso expresado en unidades arbitrarias. Los resultados se registraron en hoja de datos de formato Excel. Los datos cuantitativos son media y desviación estándar; los datos cualitativos son porcentaje de migración. El manejo de los animales se realizó de acuerdo a los Principios Éticos Internacionales y los lineamentos de la NOM-062-ZOO-1999, acerca de las "especificaciones técnicas para el cuidado y uso de animales de laboratorio". Se usaron ratas macho Wistar, de $200 \mathrm{~g}$ en promedio, de las cuales se hacen dos grupos: Grupo A) ratas control. Grupo B) ratas diabéticas (inducidas durante cuatro semanas con estreptozotocina $(65 \mathrm{mg} / \mathrm{kg}$ de peso). Posteriormente se obtuvo tejido aórtico para el estudio de la velocidad de migración; para este fin después de que las ratas fueron inducidas, se sacrificaron con una sobredosis de pentobarbital sódico administrado vía intraperitoneal $(60 \mathrm{mg} / \mathrm{kg}$ ). A continuación, se procedió a realizar una toracotomía y la aorta torácica fue extraída (desechando el cayado aórtico), y colocada en una caja de Petri, con solución salina estéril, para luego limpiar el exceso de tejido seroso y graso con instrumental fino, posteriormente se realizó un corte longitudinal la aorta y se retira el endotelio vascular raspando con ayuda de un hisopo de algodón estéril, para después cortar en segmentos de 1-2 $\mathrm{mm}^{2}$. Posteriormente, cada uno de los segmentos obtenidos se colocaron, en cajas de cultivo de $2.5 \mathrm{~cm}^{2}$ de diámetro con medio de cultivo DMEM (Dulbecco's Modified Eagle's Medium), con glucosa normal $(5 \mathrm{mM})$, suplementado con suero bovino fetal (SBF) al $10 \%$, $10000 \mathrm{U} / \mathrm{mL}$ de penicilina, $10 \mathrm{mg} / \mathrm{mL}$ de estreptomicina y colocados en la incubadora de $\mathrm{CO} 2$ al $5 \%$, a $37^{\circ} \mathrm{C}$ y humedad al $100 \%$. Se mantuvieron en la incubadora por aproximadamente 3-4 semanas para 
que las células confluyeran, realizando cambio de medio cada tercer día. Al confluir, se procedió a levantarlas en una cámara de cultivo celular estéril, usando tripsina en solución al $0.1 \%$, aplicando $3 \mathrm{~mL}$, dejando actuar entre 10-20 minutos en la incubadora, posteriormente con $7 \mathrm{~mL}$ de medio DMEM para completar un volumen de $10 \mathrm{~mL}$, se continuó con el lavado de las cajas para levantarlas; ulterior, se centrifugó el volumen obtenido por 2.5 minutos a $2.5 \times 10^{3}$; se aspiró el sobrenadante y la pastilla se re-suspendió en $10 \mathrm{~mL}$ de medio DMEM; se tomó de la anterior suspensión celular $10 \mu \mathrm{L}$ para cuantificar las células obtenidas y se transfiere a 9 cajas de Petri de $3.5 \mathrm{~cm}$ de diámetro y un stock en caja de $25 \mathrm{~cm}^{2}$ a razón de $1 \times 10^{4}$ células por caja aproximadamente. Se incubó y cambió el medio cada tercer día, con las mismas características antes mencionadas y al llegar a confluencia (1 semana después aproximadamente) se realizó el experimento de "Herida-Cicatrización" en caja de cultivo, para evaluar la velocidad de migración celular. Para fines de este reporte, se realizaron dos repeticiones del experimento de una rata control y una rata diabética. En una campana para cultivo estéril, se tomó una punta de micropipeta de $200 \mu \mathrm{L}$, y se colocaron al microscopio de luz invertido al objetivo de 10X; y se tomaron fotografías al tiempo 0, a las 12 horas y a las 24 horas. Se comparó la velocidad de migración entre las células de ratas normales cultivadas y las diabéticas. Para ello, se utilizó el programa Image J, para delimitar el área expresada en unidades arbitrarias de la herida conforme al tiempo trascurrido desde provocada la herida. Al obtener el área de ambas ratas a los distintos tiempos, en una hoja de Excel, se calculó de acuerdo al área libre y área ocupada de las dos repeticiones de rata control y diabética, el porcentaje ocupado de cada repetición, el promedio del porcentaje de migración celular y la desviación estándar del área ocupada.

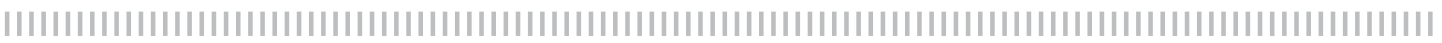

\section{Resultados}

Se realizaron dos repeticiones del experimento de una rata control (R1) y una rata diabética (R2). Se tomaron fotografías al tiempo 0 , a las 12 horas y a las 24 horas, para observar la velocidad de migración celular expresada en unidades arbitrarias que se calculó de acuerdo al área libre y área ocupada de las dos pruebas del ensayo, tanto en rata control como en rata diabética. El porcentaje de área ocupado de cada prueba a las 12 horas: la prueba 1 de rata diabética (R2) de $39.14 \%$ y prueba 2 de $55.43 \%$; a las 24 horas de $81.69 \%$ y $82.05 \%$. Mientras que de la rata control (R1) en la primera prueba a las 12 horas resultó de $9.17 \%$, en la segunda prueba de $32.17 \%$; a las 24 horas de $37 \%$ y de $66 \%$ en prueba uno y dos respectivamen- te. El registro del área libre y ocupada, así como los porcentajes de migración de las dos pruebas del experimento de rata diabética y control se encuentran en la tabla 1. El promedio y desviación estándar del porcentaje de migración celular por rata se encuentran en la tabla 2 , resultando a las 12 horas de $20.6 \%$ y $47.28 \%$, para las ratas control y diabética respectivamente y las 24 horas de $51.8 \%$ (R1) y de $81.87 \%$ (R2). De la misma manera se observan en la gráfica 1 los porcentajes de migración de acuerdo al tiempo del ensayo. La figura 2, consta de fotografías tomadas con la visión al microscopio de luz invertido al objetivo de 10X, donde se muestra la migración celular de las ratas control y diabética tras haber realizado la herida en la caja de cultivo a las 0,12 y 24 horas. 


\section{Tabla I}

Registro de los porcentajes de migración de dos repeticiones del experimento “herida-cicatrización" de rata diabética y control, a las 0, 12 y 24 horas y promedio más desviación estándar de las repeticiones de los dos grupos.

R2. Diabética 1ra prueba

R1. Control. 1ra prueba

\begin{tabular}{|c|c|c|c|c|c|c|}
\hline & 0 horas & 12 horas & 24 horas & 0 horas & 12 horas & 24 horas \\
\hline área libre & $2,707,028$ & $1,647,530$ & 495,747 & $4,078,037$ & $3,704,403$ & $2,569,323$ \\
\hline área ocupada & & $1,059,498$ & $2,211,281$ & & 373,634 & $1,508,714$ \\
\hline$\%$ ocupado & & $39.14 \%$ & $81.69 \%$ & & $9.16 \%$ & $37.00 \%$ \\
\hline \multicolumn{2}{|c|}{ R2. Diabética 2da prueba } & \multicolumn{5}{|c|}{ R1 Control. 2da prueba } \\
\hline & 0 horas & 12 horas & 24 horas & 0 horas & 12 horas & 24 horas \\
\hline área libre & 2963910 & 1321048 & 532145 & 2241614 & 1520402 & 745307 \\
\hline área ocupada & & 1642862 & 2431765 & & 721212 & 1496307 \\
\hline$\%$ ocupado & & $55.43 \%$ & $82.05 \%$ & & $32.17 \%$ & $66.75 \%$ \\
\hline Diabetes & 12 horas & 24 horas & Control & & 12 horas & 24 horas \\
\hline Promedio & $47.28 \%$ & $81.87 \%$ & Promedio & 3159825.5 & $20.67 \%$ & $51.87 \%$ \\
\hline 181643.004 & $5.76 \%$ & $0.13 \%$ & Desv. Est. & 1298547.16 & $8.14 \%$ & $10.52 \%$ \\
\hline
\end{tabular}

\section{Tabla 2}

Promedio de porcentaje de migración celular a las 12 y 24 hrs de ratas control y diabética.

\begin{tabular}{lccc} 
& 12 horas & \multicolumn{2}{c}{24 horas } \\
\hline CONTROL & $20.6 \%$ & $51.8 \%$ \\
\hline DIABÉTICA & $47.28 \%$ & $81.87 \%$ \\
\hline
\end{tabular}

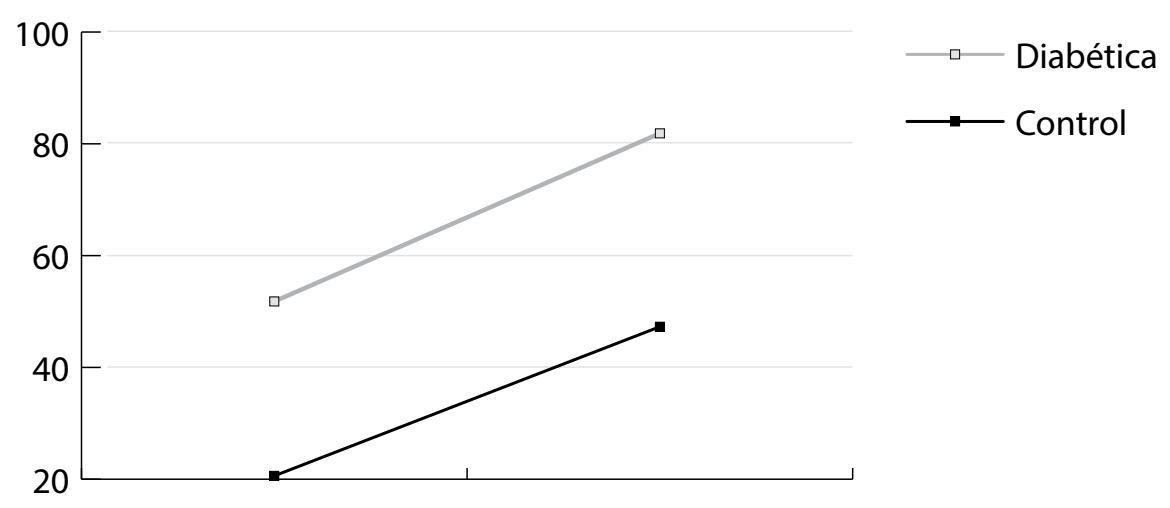

Figura 1. Gráfica donde se muestra el porcentaje de migración celular a las 12 y 24 horas de ratas control y diabética 


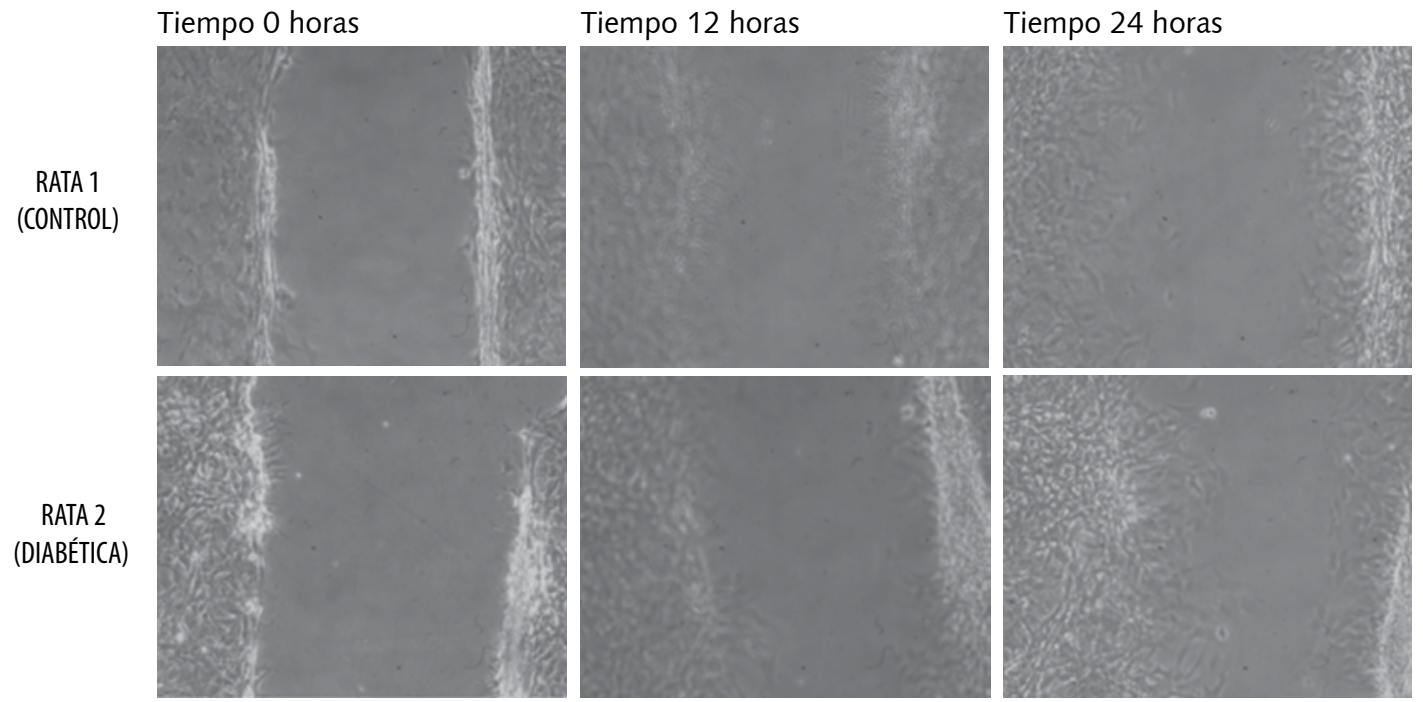

Figura 2. Fotografías donde se muestra la migración celular de las ratas control y diabética tras haber realizado la herida en la caja de cultivo, y observándose al microscopio de luz invertido al objetivo 10X, a las 0,12 y 24 horas.

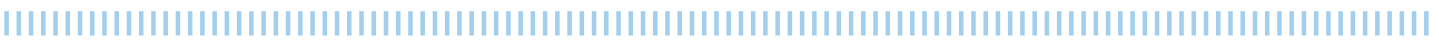

\section{Discusión}

La migración celular es un fenómeno dependiente de calcio. ${ }^{19}$ Las variaciones en las concentraciones de $\mathrm{Ca}^{2+}$ en el ambiente de las CMLV son interpretadas como señales que implican cambios en la dinámica contráctil celular. Se ha demostrado que para que las CMLV puedan migrar se requieren de aumentos transitorios de $\mathrm{Ca}^{2+}$ citosólico que se inician en el urópodo y se desplazan hacia la región frontal formando ondas de $\mathrm{Ca}^{2+}$. Estas ondas son indispensables para el proceso de migración celular. La Ca ATPasa del retículo sarcoendoplásmico (SERCA) al recibir estímulos desde la membrana celular, produce la liberación de $\mathrm{Ca}^{2+}$ por lo que este organelo parece ser el origen de las ondas de $\mathrm{Ca}^{2+}$ ya que se ha visto que la inhibición de SERCA mediante thapsigargina, abole las mencionadas ondas. ${ }^{20}$ Se ha reportado que la hiperglucemia que se observa en la DM, causa un aumento en las concentraciones de $\mathrm{Ca}^{2+}$ citosólico, produce estrés en el retículo sarcoendoplásmico (RER), llevando a disminución de la expresión de SERCA, con lo que la capacidad para almacenar $\mathrm{Ca}^{2+}$ se ve disminuida o abolida. ${ }^{17}$

En este trabajo se obtuvo como resultado una mayor velocidad de migración en las células de músculo liso vascular de la rata diabética comparada con su control. Esto contrasta con el mecanismo anterior, ya que pareciera que la DM, al condicionar estrés sobre el RER, abole el fenómeno de migración celular y por lo tanto no habría cabida al proceso de re-estenosis tras la colocación de un stent como procedimiento de revascularización debido a complicaciones macrovasculares de la DM, como la aterosclerosis. Esto llevó a postular que, en la DM, a pesar de existir el estado de estrés al RER, que disminuye la capacidad de almacenamiento de $\mathrm{Ca}^{2+}$ lo que altera las concentraciones de $\mathrm{Ca}^{2+}$ citosólico y por ende de formar ondas de $\mathrm{Ca}^{2+}$, la concentración de $\mathrm{Ca}^{2+}$ es suficiente para la excesiva migración de las CMLV. 17,21

Los resultados concuerdan con uno anterior realizado por Espinosa Tanguma 
y cols., donde compararon la velocidad de migración celular con grupos de ratas diabéticas y controles, en sus resultados observaron una mayor velocidad de migración en las ratas diabéticas en comparación con las ratas del grupo control tanto a las 12 como las 24 horas de haber realizado el ensayo de la herida en las cajas de cultivo. ${ }^{17}$ Por añadidura, se ha obtenido evidencia de que el músculo liso vascular de ratas diabéticas muestra un estado de hiperreactividad vascular, asociado a la disminución de la expresión de SERCA. ${ }^{22}$

La re-estenosis intra-stent es un problema creciente debido al importante incremento que se ha producido en la utilización del stent como forma de revascularización percutánea, su incidencia global es de alrededor del 28\%; implica complicaciones inmediatas que son similares a las que se presentan tras la implantación de un stent de novo (afectación de ramas laterales a la lesión, disecciones fuera del stent, trombosis aguda o subaguda, hemorragia), además implican un alto costo por la atención hospitalaria que demanda. Es por ello que surge la imperiosa necesidad de conocer las características cuantitativas y cualitativas de la migración celular, como lo es su velocidad y su comportamiento frente a diferentes concentraciones de glucosa en la aterosclerosis como complicación de la DM con el fin de plantear nuevas estrategias terapéuticas que reduzcan o inhiban la re-estenosis. ${ }^{23}$

\section{Conclusiones}

El fenómeno de migración de células del músculo liso es importante en los pacientes diabéticos complicados con lesiones macrovasculares como la aterosclerosis, ya que lleva a la necesidad de procedimientos de revascularización como la colocación de stents que, desafortunadamente, con el tiempo pueden presentar re-estenosis tras su colocación. Lo que lleva a la necesidad de estudiar sus características y sus factores predisponentes o causales, tales como la velocidad de migración o las variaciones en la concentración de glucosa a las que las células son sometidas con el fin de plantear nuevas medidas o estrategias terapéuticas que logren retardar o detener este proceso dependiente de $\mathrm{Ca} 2+$ y que impone una problemática socio sanitario creciente.

\section{Bibliografía}

1. Diagnosis and Classification of Diabetes Mellitus. Diabetes Care. enero de 2010;33(Suppl 1):S62-9.

2. Forouhi NG, Wareham NJ. Epidemiology of diabetes. Med Abingdon Engl UK Ed. diciembre de 2014;42(12):698-702.

3. Hill J, Nielsen $M$, Fox $M H$. Understanding the Social Factors That Contribute to Diabetes: A Means to Informing Health Care and Social Policies for the Chronically III. Perm J. 2013;17(2):67-72.

4. Henry WL. The Complications of Diabetes Mellitus. J Natl Med Assoc. junio de 1987;79(6):677-80.

5. Beckman JA, Creager MA, Libby P. Diabetes and atherosclerosis: epidemiology, pathophysiolo- gy, and management. JAMA. el 15 de mayo de 2002;287(19):2570-81.

6. Centers for Disease and Prevention. National Diabetes statistics Report: Estimates of Diabetes and its Burden in the United States. 2014;

7. Control TD, Group CTDR. Effect of intensive diabetes management on macrovascular events and risk factors in the diabetes control and complications trial. Am J Cardiol. 1995;75(14):894-903.

8. Kastrati $A$, Schühlen $H$, Hausleiter J, Walter $H$, Zitzmann-Roth $E$, Hadamitzky $M$, et al. Restenosis after coronary stent placement and randomization to a 4-week combined antiplatelet or anticoagulant therapy: six-month angiographic follow-up of the Intracoronary Stenting and Antithrombotic Regimen 
(ISAR) Trial. Circulation. 1997:96(2):462-467.

9. Kuntz RE, Gibson CM, Nobuyoshi M, Baim DS. Generalized model of restenosis after conventional balloon angioplasty, stenting and directional atherectomy. J Am Coll Cardiol. 1993;21(1):15-25.

10. Huang $B$, Dreyer $T$, Heidt $M$, Julie $C M$, Philipp $M$, Hehrlein FW, et al. Insulin and local growth factor PDGF induce intimal hyperplasia in bypass graft culture models of saphenous vein and internal mammary artery. Eur J Cardiothorac Surg. 2002;21(6):1002-1008.

11. Farb A, Weber DK, Kolodgie FD, Burke AP, Virmani R. Morphological predictors of restenosis after coronary stenting in humans. Circulation. 2002;105(25):2974-2980.

12. Faries $\mathrm{PL}$, Rohan $\mathrm{DI}$, Wyers $M C$, Marin $\mathrm{ML}$, Hollier $\mathrm{LH}$, Quist WC, et al. Relationship of the $20 \mathrm{~S}$ proteasome and the proteasome activator PA28 to atherosclerosis and intimal hyperplasia in the human vascular system. Ann Vasc Surg. 2001;15(6):628-633.

13. Madi HA, Riches K, Warburton P, O'Regan DJ, Turner NA, Porter KE. Inherent differences in morphology, proliferation, and migration in saphenous vein smooth muscle cells cultured from nondiabetic and Type 2 diabetic patients. Am J Physiol-Cell Physiol. 2009;297(5):C1307-C1317.

14. Gerthoffer WT. Mechanisms of vascular smooth muscle cell migration. Circ Res. 2007;100(5):607621.

15. Yamaguchi $N$, Anand-Apte $B$, Lee $M$, Sasaki $T$, Fukai $N$, Shapiro $R$, et al. Endostatin inhibits VEGF-induced endothelial cell migration and tumor growth independently of zinc binding. EMBO J. 1999;18(16):4414-4423.

16. Fay FS, Gilbert SH, Brundage RA. Calcium signalling during chemotaxis. En: Calcium waves, gradients and oscillations Ciba Foundation Symposium [Inter- net]. 1995 [citado el 1 de agosto de 2016]. p. 121140. Disponible en: https://books.google.com.mx/ books?hl=es\&lr =\&id=hsxpwF7umhMC\&oi $=$ fnd \& $\mathrm{pg}=\mathrm{PA} 121 \& \mathrm{dq}=\mathrm{RODNEY}+\mathrm{A}+\mathrm{BRUNDAGE}+1999$ + CELLULAR + MIGRATION\&ots $=$ UCMO-5ytJH\&si $\mathrm{g}=$ QwOPSYBtvKYPfsKD3DyKEHftpyM

17. Espinosa-Tanguma $R$, Caroline $O$, Chrones T, Pickering JG, Sims SM, others. Essential role for calcium waves in migration of human vascular smooth muscle cells. Am J Physiol-Heart Circ Physiol. 2011;301(2):H315-H323.

18. Wei $C$, Wang $X$, Zheng $M$, Cheng $H$. Calcium gradients underlying cell migration. Curr Opin Cell Biol. 2012;24(2):254-261.

19. Marks PW, Maxfield FR. Local and global changes in cytosolic free calcium in neutrophils during chemotaxis and phagocytosis. Cell Calcium. 1990;11(2):181190.

20. Cavarra MS, Assef YA, Kotsias BA. Effects of ionomycin and thapsigargin on ion currents in oocytes of Bufo arenarum. J Exp Zoolog A Comp Exp Biol. 2003;297(2):130-137.

21. Gardel ML, Schneider IC, Aratyn-Schaus $Y$, Waterman CM. Mechanical integration of actin and adhesion dynamics in cell migration. Annu Rev Cell Dev Biol. 2010;26:315.

22. Obrosova IG, Minchenko AG, Marinescu $V$, FathaIlah $L$, Kennedy A, Stockert CM, et al. Antioxidants attenuate early up regulation of retinal vascular endothelial growth factor in streptozotocin-diabetic rats. Diabetologia. 2001;44(9):1102-1110.

23. Gómez-Hospital JA, Cequier Á, Fernández-Nofrerías E, Mauri J, del Blanco BG, Iráculis $E$, et al. Tratamiento de la reestenosis intra-stent. Situación actual y perspectivas futuras. Rev Esp Cardiol. 1999;52(12):1130. 